\title{
Student Difficulties With Trigonometric Vector Components Persist In Multiple Student Populations
}

\author{
Brendon D. Mikula and Andrew F. Heckler \\ Department of Physics, The Ohio State University, Columbus, OH 43210
}

\begin{abstract}
Students in introductory physics courses sometimes struggle to correctly break down a single vector into its components when provided only with an arrow, a magnitude, a reference angle, and a coordinate system. Students struggle further when asked to break down a vector in an inclined coordinate system, such as the weight vector of a box on an inclined plane. Varying the placement of the angle consistently affects student error and response patterns across four physics student populations: algebra-based mechanics, algebra-based E\&M, calculus-based mechanics, and calculusbased E\&M. This suggests that student difficulties with trigonometric vector components are persistent and pervasive, even across different introductory physics courses, and are far below the requisite near-perfect accuracy needed for such fundamental skills. Student error and response patterns are discussed for both problem types.
\end{abstract}

Keywords: Student understanding, physics education research, vectors, vector components, trigonometry.

PACS: $01.55 .+\mathrm{b}, 01.40 .-\mathrm{d}$

\section{INTRODUCTION}

An integral step in many physics problems is the application of trigonometric functions to analyze vectors in terms of perpendicular components. The skill itself is fundamental and essential knowledge for introductory physics. As such, it is imperative that students are as close to $100 \%$ accuracy as possible at trigonometric vector decomposition. However, as we will show in this study, a significant number of students have specific difficulties even with the simplest of trigonometric vector problems.

Previous studies on student understanding of vector components and decomposition have found significant student difficulties. For example, Van Deventer and Wittmann found that only $54 \%$ of students in a large U.S. public university were able to select the correct magnitude of a trigonometric vector component when given multiple-choice options to choose from [1]. In a related study about components of vectors on a gridnot using trig functions-Zavala and Barniol showed that only $\sim 85 \%$ of introductory physics students at a private Mexican university were able to correctly choose the components of a vector presented on a grid [2]. Aguirre and Rankin found that only about $50 \%$ of students understood the vector nature of velocity in terms of orthogonal components [3]. Finally, Knight found that less than half of students could calculate the magnitude and direction (i.e. angle from $x$-axis) of a vector presented on a grid or in $i-j-k$ format [4].

While these studies provide valuable insight into a number of student difficulties with simple vector problems, this study focuses on the investigation of student difficulties with the use of trigonometric functions on simple vector decomposition problems. While the study of these difficulties is interesting in its own right, the results may also be useful for the improvement of instruction on this important skill.

\section{PARTICIPANTS AND DESIGN}

Data was collected after relevant instruction over three separate terms from four introductory physics student populations: algebra-based mechanics $(\mathrm{N}=$ 636), algebra-based $\mathrm{E} \& \mathrm{M}(\mathrm{N}=168)$, calculus-based mechanics $(\mathrm{N}=229)$, and calculus-based $\mathrm{E} \& \mathrm{M}(\mathrm{N}=$ 188). Data is presented for all four populations.

In order to study the effects of angle placement on student aptitude with trigonometric vector components, we constructed a task consisting of three problems (Fig. 1). Participants were randomly assigned to one of four conditions, each consisting of two "simple" problems and one inclined plane problem. The specific questions in each condition are shown in Table 1.

TABLE 1. Question makeup of the four conditions. H and $\mathrm{V}$ (Horiz and Vert) designate whether the angle is given with respect to the horizontal or the vertical. $\mathrm{X}$ and $\mathrm{Y}$ designate which of the two components was requested. For simple problems, Tip and Tail designate whether the angle is given with respect to the tip or tail of the vector.

\begin{tabular}{|l|l|l|l|}
\hline & \multicolumn{2}{|c|}{ (Simple) } & \multicolumn{1}{c|}{ (Incline) } \\
& \multicolumn{1}{|c|}{ Q1 } & \multicolumn{1}{c|}{ Q3 } \\
\hline Condition 1 & H Tail X & H Tip X & Horiz X \\
\hline Condition 2 & H Tail Y & H Tip Y & Vert X \\
\hline Condition 3 & V Tail X & V Tip X & Vert Y \\
\hline Condition 4 & V Tail Y & V Tip Y & Horiz Y \\
\hline
\end{tabular}



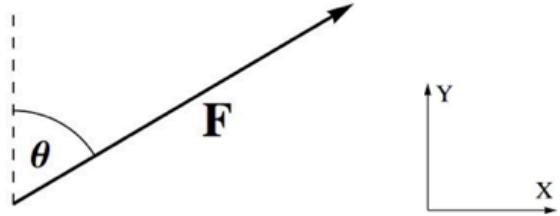

1. A vector with magnitude $F$ is shown. Find its component in the $x$ direction.
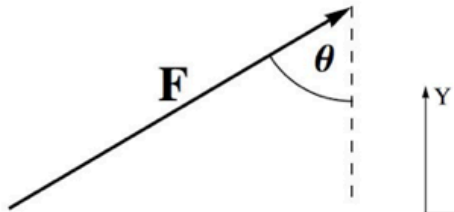

$\stackrel{\mathrm{X}}{\longrightarrow}$

2. A vector with magnitude $F$ is shown. Find its component in the $x$ direction.

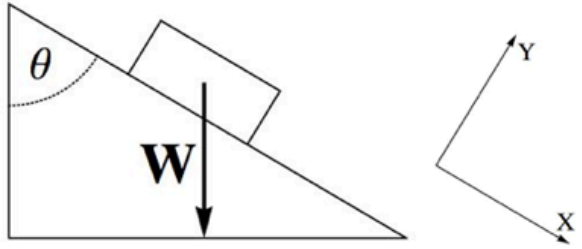

3. A block with weight of magnitude $W$ sits at rest on an inclined plane, as shown. Find the component of the weight vector $W$ in the $y$ direction.

FIGURE 1. The three problems from one of the four experimental conditions (condition 3 is shown above). Each student received two simple problems $(\# 1,2)$ and one inclined plane problem (\#3). Both problem types varied angle placement (from horiz./vert.) and which component ( $x$ or $y$ ) was requested between conditions. Simple problems varied angle given from the tip/tail of the vector within condition (see Table 1). All problems were accompanied by the note "NOTE: $X$ and $Y$ directions are positive in the directions indicated by the coordinate axes. Please answer in terms of given magnitudes and trig functions of given angles."

\section{Coding of Student Responses}

Each problem was coded for correctness as well as for specific student response patterns and errors. A list of descriptions for each coding category follows:

- Correct: Student response is identical or mathematically equivalent to the correct answer.

- Trig Fxn: The trigonometric function included in the student response is correct, even if the other errorssuch as a stray negative sign-were present.

- CTF: Complementary trig function is used, e.g. $\mathrm{F}^{*} \cos \left(90^{\circ}-\theta\right)$ instead of $\mathrm{F}^{*} \sin (\theta)$.

- Sin/Cos Error: Student switches sine and cosine, effectively answering for the wrong component.

- Sign Error: Student response differs from correct response in terms of sign (positive or negative).

- Wrong Triangle Error (only for inclined plane): Student answers based on a triangle in which the weight vector is not the hypotenuse. This error yields answers containing $\tan (\theta), \cot (\theta), \sec (\theta)$, and $\csc (\theta)$.

- Wrong Angle Error (only for inclined plane): When students generated angles on the question diagram, the geometric correctness of the angle marked was noted. Results for this error are presented separately.

Unless noted above, all problems were coded for all categories of student responses. Only categories with significant response rates are discussed in this paper. Due to small and inconsistent differences between Mechanics and E\&M, this category was collapsed during most of analysis.

Unless noted, statistics were performed using a Generalized Linear Model (GLM) w/ a Binary Logistic dependent variable and Wald $\chi^{2}$ values are reported.

\section{RESULTS AND DISCUSSION}

\section{Simple Problems}

No differences were found between $x$ and $y$ components for the first two "simple" problems, so this category was collapsed during analysis.

Figure 2 presents the score for the four angle configurations for the simple problems. Students performed best $(80-90 \%$ correct) when the angle was given from the tail of the vector with respect to the horizontal. Accuracy was lowest $(60-70 \%)$ when the angle was given with respect to the horizontal from the tip of the vector and intermediate when the angle was given with respect to the vertical. For each angle configuration, students in the calculus-based sequence outperformed their algebra-based counterparts. It is worth noting that these all represent post-instruction results. Given the fundamental nature of this simple task, the distances from ceiling are troubling.

Specific student response patterns and errors also showed dependence on angle configuration (Fig. 3).

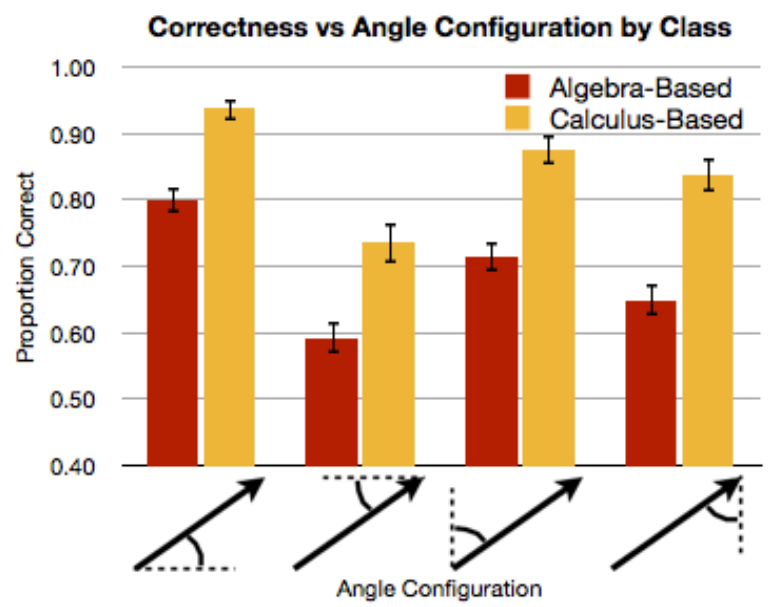

FIGURE 2. Proportion of correct responses for each angle configuration on simple problems. Angle configurations are represented pictorially along the horizontal axis of the figure. All differences between algebra and calculus-based populations are statistically significant $(\alpha=0.05)$. 


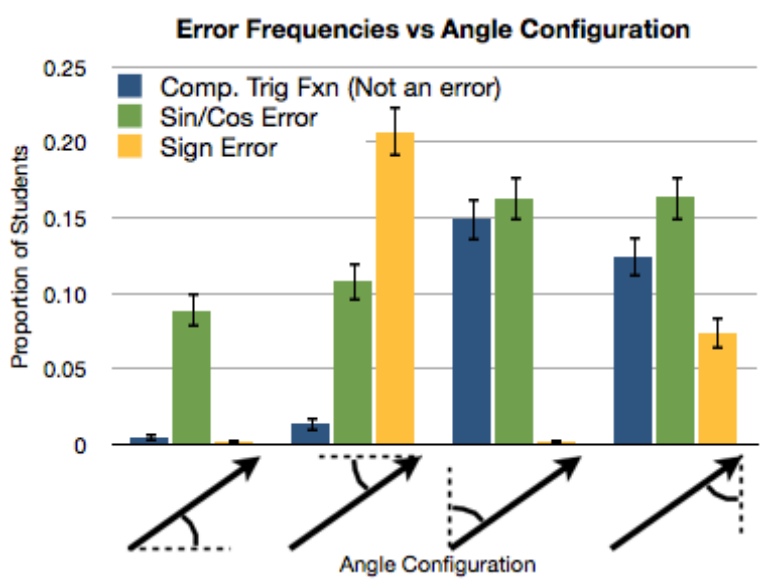

FIGURE 3. Student response and error patterns for each angle configuration. Note that Complementary Trig Function use is not an error, but an alternate solution path. Complementary Trig Function use and $\mathrm{Sin} / \mathrm{Cos}$ errors are more common when the angle is given from the vertical. Sign errors are almost exclusive to angles being given from the tip of a vector.

Students used complementary trig functions more frequently when the angle was given from vertical $\left(\chi^{2}(1, \mathrm{~N}=2388)=81.7, p<0.001\right)$, suggesting that students are most familiar with angles given from horizontal and attempt to transform problems into this familiar form. The primary errors were sine-cosine confusion and a sign error. A 2-factor GLM (horiz.vert. vs. tip-tail) revealed that $\mathrm{Sin} / \mathrm{Cos}$ errors occurred more frequently for the vertical angle $\left(\chi^{2}(1, N=2388)=\right.$ $21.9, p<0.001)$, suggesting that students may not be familiar with both angle configurations and may need more practice on problems drawing attention to the

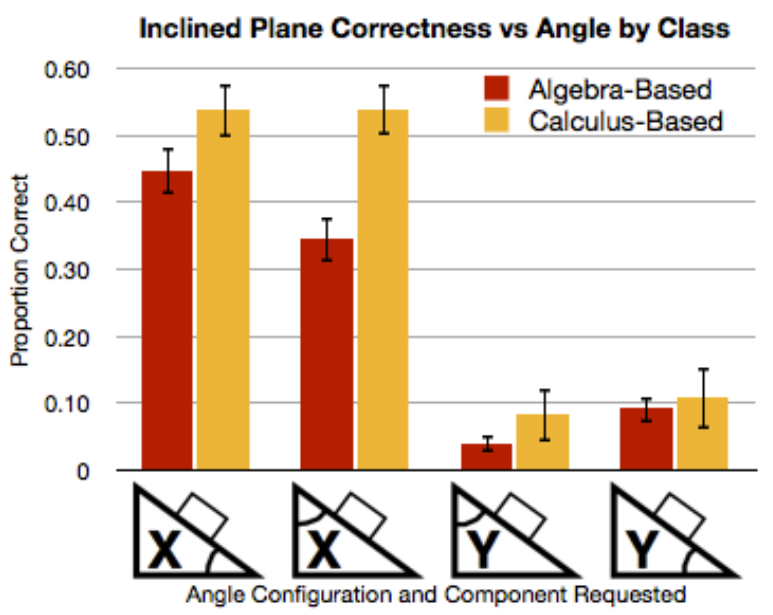

FIGURE 4. Proportion of correct responses for each angle configuration on inclined plane problems. Angle configurations and component requested are represented pictorially along the horizontal axis of the figure. The only significant difference between algebra and calculus-based students was when the angle was given from the vertical and the $x$ component was requested $(\alpha=0.05)$. contrast. No other interaction or main effects were significant for the $\mathrm{Sin} / \mathrm{Cos}$ error.

Sign errors were committed almost exclusively when the angle was given from the tip of the vector $\left(\chi^{2}(1, \mathrm{~N}=2442)=41.8, p<0.001\right)$. This suggests that the angle configuration may be the more salient cue for vector component direction with some students than the direction of the arrow itself. Paraphrasing, when given an angle from the tip, students seem to be treating the vector as though it were in the $3^{\text {rd }}$ quadrant.

\section{Inclined Plane Problems}

For inclined plane problems, students performed best when the $x$ component was requested, regardless of whether the angle was given with respect to the horizontal or the vertical (Fig. 4). However, even the best scores in the highest-performing populations are barely above $50 \%$; student responses to $y$ component problems are about $10 \%$ correct or worse.

Figure 5 presents specific student response patterns. Sign errors occurred frequently and almost exclusively when the $y$ component was requested $\left(\chi^{2}(1, \mathrm{~N}=1132)=\right.$ $368, p<0.001)$, accounting for almost all of the score difference between the two components. The error could be aggravated by the fact that sign errors on $x$ component problems represented the erroneous inclusion of a negative sign, where sign errors on $y$ component problems represented a missing negative sign that should have been included.

Students found the correct trig function approximately the same proportion of time, regardless of which component was requested. However, a 2factor GLM ( $x-y$ vs. angle placement) revealed that the use of correct trig functions significantly depended on angle placement $\left(\chi^{2}(1, \mathrm{~N}=1133)=5.16, p=0.023\right)$.

A similar 2-factor GLM on $\mathrm{Sin} / \mathrm{Cos}$ errors also showed significant dependence on angle placement $\left(\chi^{2}(1, \mathrm{~N}=1133)=6.76, p=0.009\right)$ with more errors for the vertical angle. This suggests that $\sim 70 \%$ of students are actually working through — not just memorizingthe application of trig functions to these problems. However, a significant proportion of students appear to be confusing sine and cosine or simply using a memorized algorithm based on the common horizontal angle. Part of the Sin/Cos error signal could come from students labeling an incorrect angle in on the question diagram; this will be discussed in depth below.

Wrong triangle errors - answering based on a triangle containing the weight vector as a leg instead of the hypotenuse - showed no significant dependence on angle placement or component requested. This suggests that a small, but consistent, proportion of students fail to follow even the most basic rules of setting up triangles to break vectors into components. 


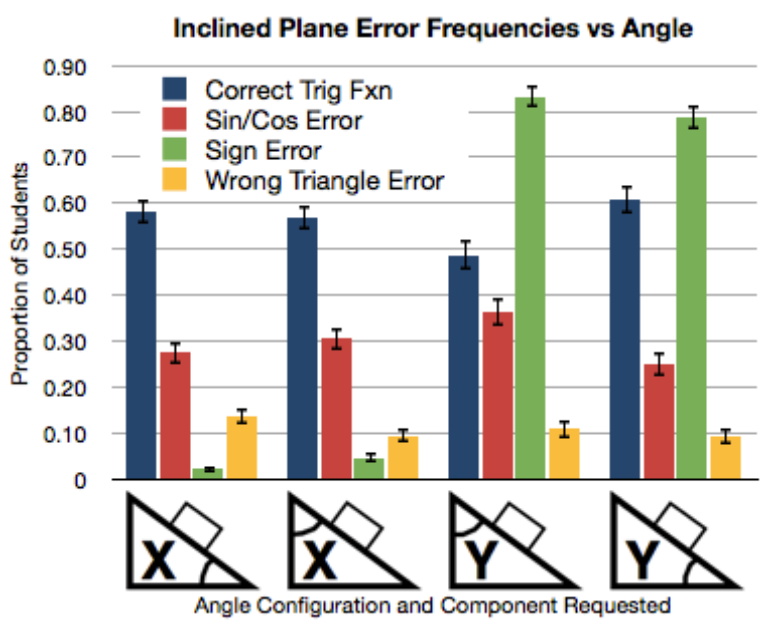

FIGURE 5. Student response and error patterns in inclined plane problems for each angle configuration. Note that Correct Trig Fxn is not an error, but a useful metric to factor out the influence of sign errors. Sign errors were committed almost exclusively when the $y$ component was requested. Wrong angle errors were also coded, but are included separately below as not all students drew angles on the provided question diagram.

Some students indicated given angles on the diagram presented with the question. These were coded for geometrical correctness and consistency with the trig function in the student's solution. As shown in Table 2, 65\% (50\%) of calculus (algebra) students drew an angle on the diagram, and $81 \%(73 \%)$ of these students indicated the correct angle. Correspondingly, $83 \%(79 \%)$ of these students submitted a response consistent with the angle marked, suggesting that students may be slightly better with trigonometry than with geometry. Notably, calculus (algebra) students who indicated an angle were about $22 \%$ (17\%) more likely to choose the correct trig function than those who indicated no angle, regardless of whether the angle indicated was correct $\left(\chi_{\text {Pearson }}^{2}(d f=1,1 ; N=804\right.$, $417)=24.3,20.0 ; p<0.001,0.001 ; d=0.36,0.47 ; \phi_{C}$ $=0.857,0.979$; respectively). This provides evidence that the act of marking up the angles on the provided question diagram improves accuracy.

TABLE 2. Summary of student-drawn angles and responses on inclined plane problems, split into algebra and calculus-based populations. Asterisks indicate statistical significance at the $\alpha=0.05$ level.

\begin{tabular}{|l|l|l|}
\hline & Algebra & Calculus \\
\hline Drew an angle & $50 \%$ & $65 \%$ \\
\hline $\begin{array}{c}\text { Drew correct angle } \\
\text { (given angle drawn) }\end{array}$ & $73 \%$ & $81 \%$ \\
\hline $\begin{array}{c}\text { Angle and answer consistent } \\
\text { (given angle drawn) }\end{array}$ & $79 \%$ & $83 \%$ \\
\hline $\begin{array}{c}\text { Correct Trig Function } \\
\text { (given NO angle drawn) }\end{array}$ & $44 \%$ & $49 \%$ \\
\hline $\begin{array}{c}\text { Correct Trig Function } \\
\text { (given angle drawn) }\end{array}$ & $61 \%$ & $71 \%$ \\
\cline { 3 - 4 }
\end{tabular}

\section{CONCLUSION \& IMPLICATIONS FOR INSTRUCTION}

We found that, even post instruction, student performance is nowhere near the $90-100 \%$ accuracy needed for the essential skill of applying trigonometric functions to vector decomposition. Students often confuse sine with cosine when the angle is given from vertical and commit sign errors when the angle is given from the tip of the vector. On inclined plane problems, students frequently omit necessary signs, confuse sine with cosine, and answer based on incorrectly drawn triangles and incorrectly placed angles. Overall, the results suggest that students often produce answers based on the most commonly seen configuration-i.e. angle taken from the horizontal axis, as seen in the unit circle and polar coordinates - regardless of where the reference angle is placed.

While the fact that students struggle with the basic application of trigonometric functions may be familiar to many instructors, this study documents the extent of the problem and carefully characterizes the nature of the difficulties in order to highlight the need for, and suggest avenues to pursue, improving this essential skill. Clearly students need more practice on a variety of angle configurations. Of special concern are wrong triangle errors, which represent a fundamental failure to understand the most basic concepts of geometry and vector components. Instructional time and student practice spent on vector components-especially for inclined plane problems - should emphasize the correct way to draw triangles to aid in trigonometric vector break-down. Lastly, simply attempting to draw an angle on the question diagram-regardless of the geometrical correctness of the angle-is correlated with higher scores. This consequence of marking up the question statement with relevant information could help convince the students of the utility of such practice.

\section{ACKNOWLEDGMENTS}

This work has been supported in part by the Center for Emergent Materials at the Ohio State University, an NSF MRSEC (Award Number DMR-0820414).

\section{REFERENCES}

1. J. Van Deventer \& M. C. Wittmann, AIP Conference Proceedings, 951, 208-211 (2007).

2. G. Zavala \& P. Barniol, AIP Conference Proceedings, 1289, 341-344 (2010).

3. J. M. Aguirre \& G. Rankin, Physics Education, 24(5), 290-294 (1989).

4. R. D. Knight, The Physics Teacher, 33(2), 74-77 (1995). 